\title{
Free Re-boost Electrodynamic Tether on the International Space Station
}

\author{
Joseph A. Bonometti ${ }^{*}$ and Kirk F. Sorensen ${ }^{\dagger}$ \\ NASA - Marshall Space Flight Center, Huntsville, AL 35812 \\ Ralph H Jansen ${ }^{\ddagger}$ \\ University of Toledo, $\mathrm{OH}, 43606$
}

John W. Dankanich ${ }^{\S}$ and Kyle L. Frame**

Gray Research, Inc., Huntsville, AL, 35806

\begin{abstract}
The International Space Station (ISS) currently experiences significant orbital drag that requires constant make up propulsion or the Station will quickly reenter the Earth's Atmosphere. The reboost propulsion is presently achieved through the firing of hydrazine rockets at the cost of considerable propellant mass. The problem will inevitably grow much worse as station components continue to be assembled, particularly when the full solar panel arrays are deployed. This paper discusses many long established themes on electrodynamic propulsion in the context of Exploration relevance, shows how to couple unique ISS electrical power system characteristics and suggests a way to tremendously impact ISS's sustainability. Besides allowing launch mass and volume presently reserved for reboost propellant to be reallocated for science experiments and other critically needed supplies, there are a series of technology hardware demonstrations steps that can be accomplished on ISS, which are helpful to NASA's Exploration mission. The suggested ElectroDynamic (ED) tether and flywheel approach is distinctive in its use of 'free' energy currently unusable, yet presently available from the existing solar array panels on ISS. The ideas presented are intended to maximize the utility of Station and radically increase orbital safety.
\end{abstract}

\section{Introduction}

$\mathrm{N}$ umerous studies and proposals have clearly shown tethers in their many various forms, be they electrodynamic, momentum-exchange, structural cables, astronaut safety lines or other functions, have great benefits in space applications. The National Commission on Space (NCOS) of 1985, included Neal Armstrong, Charles Yeager, and many other prominent figures who specifically stated, "The Commission recommends that: Tether demonstrations be carried out in space both on shuttle missions and as an integral part of the initiol Space Station research proğrum.":!

The drag profile history of the ISS is clear empirical support for the case made in many system-level studies to reboost large space assets with ED tether propulsion. Figure 1 shows the altitude data readily available from the internet of the existing ISS components in orbit. Some 20 reboost propulsive burns were required during the first 4 years of operations. This is considerable when noting that only a small fraction of the overall facility was in orbit during that time. After losing the Space Shuttle Columbia, it appears that the solar arrays, the major contributor to the drag component, have been maintained into the ram direction (i.e., tangent to the orbital motion) to minimize the cross-sectional area. This helped reduce the time between propulsive reboost burns, but diminished the solar power collection because the arrays were no longer tracking the sun. Consequently, ISS is now deficient in electrical

\footnotetext{
•Emerging Propulsion Technologies Manager, In-Space Propulsion Technology Office, NP40, Joseph.A.Bonometti@nasa.gov, Member, AIAA.

${ }^{\dagger}$ Lead Systems Engineer, In-Space Propulsion Technology Office, NP23, Kirk.F.Sorensen@nasa.gov, Member, AIAA.

‡ Senior Research Associate, Mechanical, Industrial, and Manufacturing Engineering, Ralph.H.Jansen@nasa.gov, Member, AIAA.

$\$$ Aerospace Engineer, In-Space Propulsion, John.Dankanich@msfc.nasa.gov, Member, AIAA.

** Project Support, In-Space Propulsion, Kyle.L.Frame@msfc.nasa.gov, Member, AIAA.
}

This material is declared a work of the U.S. Government and is not subject to copyright protection in the United States 
power, dependent on one foreign launch vehicle for propellant resupply, disruptive to the microgravity environments during reboost operations, and risks reentry in the case that resupply is interrupted for any extended period of time.

There are two other Station subsystems that are vulnerable, yet presently offer an excellent opportunity for modern technical solutions to be demonstrated. First, the ISS batteries and their charging system have unique characteristics that prevent all the solar array power from being fully utilized. But a flywheel energy storage system replacement, a more efficient technology in its own right, could take advantage of this 'free' energy. Secondly, the Control Moment Gyros (CMGs) constantly balance the inherently unstable ISS facility. Both of these systems could be supported by the introduction of a reboost ElectroDynamic Tether (EDT) system.

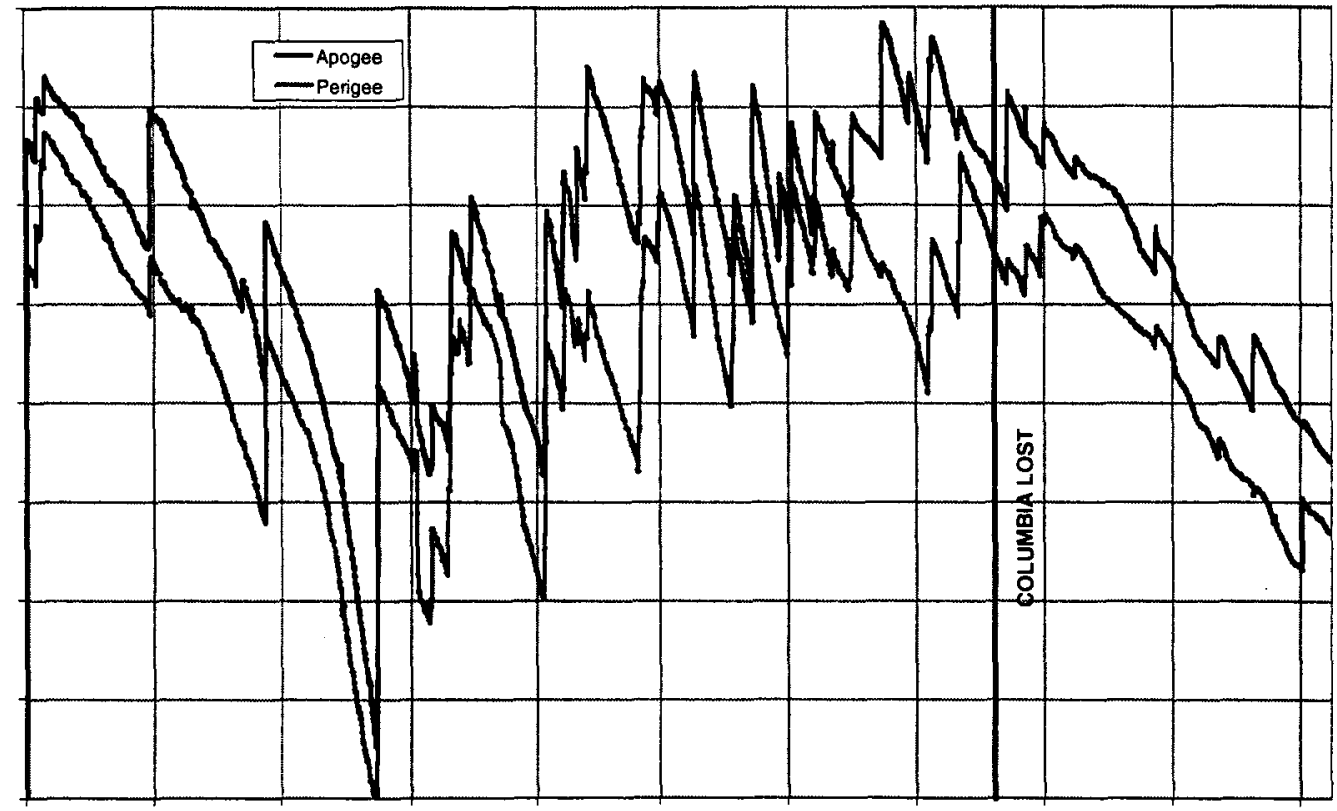

Figure 1. ISS Altitude History 1998-2004

\section{Battery Technology Limitation Affords 'Free' Propulsive Power}

Batteries have historically been used to provide energy storage for satellites as well as for other space applications. However, they are very limited in how they can be charged and discharged in both depth and duration and are sensitive to the frequency and magnitude of the peak loads. Battery technologies are inherently heavy and offer little chance of any step-function improvement in performance in future models. Voltages are nominally very low and system flexibly is limited; impacting power and distribution architectures mass and efficiencies. Dormancy for long periods will often have an adverse effect on useful lifetime. Furthermore, many battery systems require precise temperature control. Nickel hydrogen $(\mathrm{NiH})$ batteries need to be controlled within a $0-10^{\circ} \mathrm{C}$ range, while the slightly more robust Lithium ion batteries can operate between $10-30^{\circ} \mathrm{C}$. Since typical space battery applications also need a separate set of gyro wheels to maintain satellite attitude control, their overall energy density in these types of applications is about $7 \mathrm{~W}-\mathrm{hr} / \mathrm{kg}$ at the spacecraft system level. The specific ISS application likely is worse, considering the technology, structure, man-rating and other implications its unstable configuration imposes.

In applications where the energy storage system sees many thousands of charge/discharge cycles over its lifetime, the batteries, to have any reasonable lifetime, need to limit their depth of discharge as well as their rate of charge/discharge. They also need to be taper-charged during the end of any isolation period to top the battery off to

This material is declared a work of the U.S. Government and is not subject to copyright protection in the United States 
the full-charged condition. This taper-charging procedure, as presently engineered on the International Space Station, discards a great deal of solar power collected at the solar panels. As can be seen in Fig. 2, the excess solar array capacity over 13 minutes is nearly two kilowatts in this nominal orbit example. The power is actually wasted at the solar panel level by being shorted when it is not being used and simply adds heat into the solar array. This

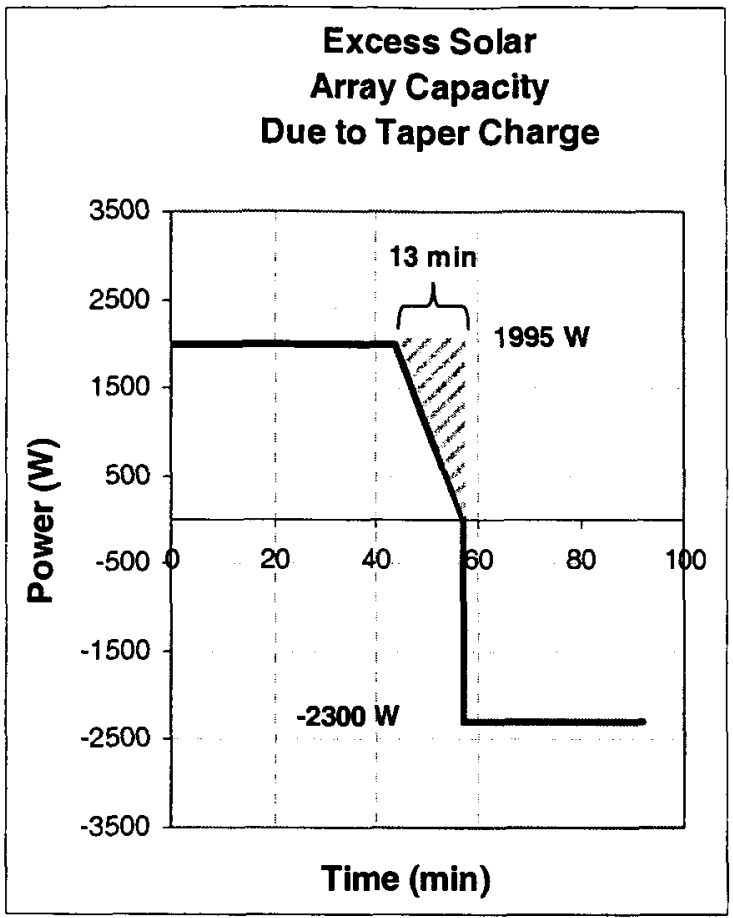

Figure 2. ISS “Free” Energy

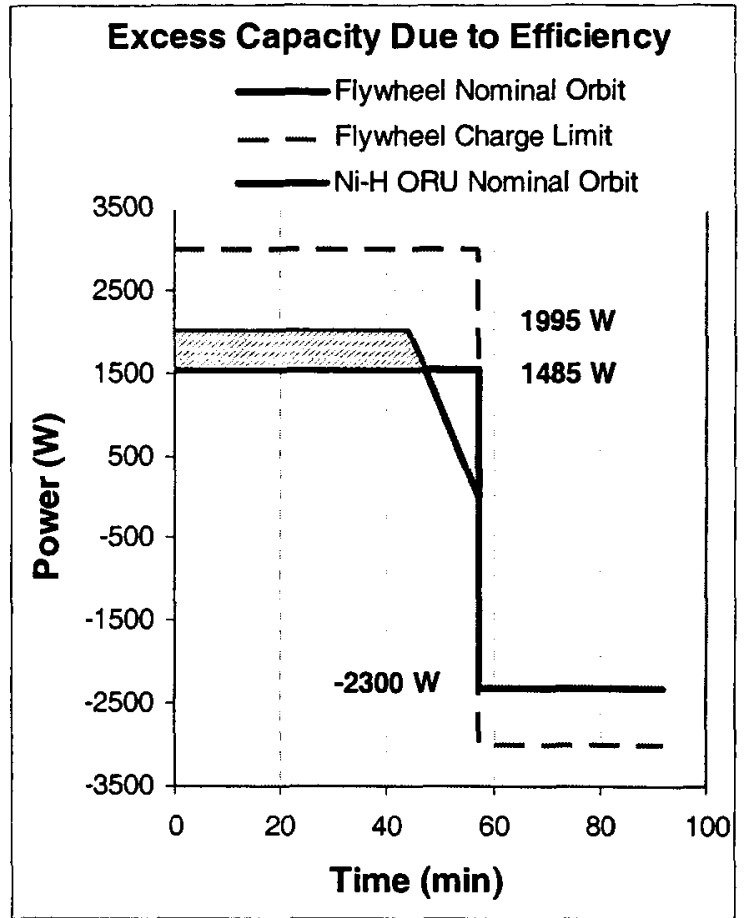

Figure 3. Combined Taper Charge/Efficiency

unused and thus 'free' energy can be tapped for propulsive use if a proper power storage system is available to take advantage of the battery system's charging restrictions. Flywheel power storage technology is capable of achieving this and is the first critical component to an ED reboost experiment and other innovative new capabilities aboard ISS. Flywheel technology also offers substantially greater power system benefits over electrochemical batteries in most future spaceflight applications.

\section{Flywheel's Unique Advantages}

Glenn Research Center (GRC) has assessed the impact of eventually converting the energy storage system for ISS fiom $\mathrm{Ni}-\mathrm{H}$ to fiywheeis. Upgrading the entire ISS energy storage system with flywheels can provide significant additional power for user loads as well as other operational and cost benefits. But even a limited infusion of flywheel replacements would have immediate beneficial impacts. Flywheels have a flexible charge profile, which allows utilization of the solar array power during the period when the $\mathrm{Ni}-\mathrm{H}$ batteries are being taper charged. For a nominal orbit, the taper charge period is thirteen minutes during which the charge power ramps down from $1995 \mathrm{~W}$ to $0 \mathrm{~W}$ per battery ORU (Fig. 1). This equates to $215 \mathrm{~W}$-hr of wasted energy per battery ORU.

Another energy savings results from the superior roundtrip efficiency of the flywheel system compared to the $\mathrm{Ni}-\mathrm{H}$ system. Roundtrip efficiency is the product of the efficiency of the electronics in charge and discharge and the efficiency of the energy storage device in charge and discharge. The nominal efficiency for the Battery Charge / Discharge Unit (BCDU) is $94 \%$ and drops off during the taper charge period. The ratio of the specified charge to discharge energy of the Ni-H ORU $(1677,1342 \mathrm{~W}$-hr) can be converted into an average battery charge and discharge efficiency of $89 \%$. The roundtrip efficiency of the battery system in a nominal orbit is $70 \%$. Battery efficiency can be greatly affected by the operating profile and other factors, however a nominal orbit was chosen for comparison purposes. The flywheel power electronics are estimated at $95 \%$ efficiency based on current technology in use by spacecraft prime contractors. The flywheel module is estimated to be $97 \%$ efficient in charge and discharge. The roundtrip efficiency of the flywheel system for a nominal orbit is $85 \%$. Higher efficiency reduces the flywheel charging power required to produce the same amount of output energy. For a nominal orbit the charge

This material is declared a work of the U.S. Government and is not subject to copyright protection in the United States 
rate, if no taper period were required for the battery, would be $1765 \mathrm{~W}$ compared to $1485 \mathrm{~W}$ for a flywheel for a savings of $265 \mathrm{~W}$-hr per ORU. Figure 3 shows the combination of the taper charge and efficiency benefits at the ORU level.

Translating the benefits gained at the ORU level back to the input of the BCDU results in a nominal charge rate per battery of $2120 \mathrm{~W}$ compared to $1560 \mathrm{~W}$ for a flywheel. For the EDT case, the initial flywheels installed would save more than enough power to operate all the subsystems associated with the reboost tether system. At the extreme end, a station-complete system with 48 ORUs deployment of flywheels will result in $25.5 \mathrm{~kW}$-hr of extra capacity during insulation. Some of this capacity could be used during the isolation period if the flywheel energy storage system was designed with extra capacity or it could be sent to user loads. The amount of power available to the user depends on several implementation specific factors, but assuming $80 \%$ efficiency through the remaining Electrical Power System (EPS) elements would result in $20 \mathrm{~kW}$-hr of extra user power capacity per orbit.

\section{Previous Tether Applications To ISS}

Using an EDT as a propulsive mechanism for ISS was investigated by C. Les Johnson in the mid-1990s $\mathrm{s}^{2,3}$. The initial design was a downward-deployed TSS-derived tether with length of $\sim 7$ kilometers. The proposal was simple and inexpensive to develop for the tether subsystem, but required tapping into Station's power and utilizing the existing plasma contactors. At the time, electric propulsion engines using propellant were being studied for the same reboost role, but all had significant drawbacks ${ }^{4}$. Although the Tether Reboost System (TRS) proposed by Johnson offered great savings over any electric thruster, this design, along with various other tether designs that soon followed, was not embraced due to the purpose and setup of Station. At the time, ISS could not use a tether for propulsion, since a tether would significantly shift the CM and pose a hindrance to the onboard microgravity experiments, interfere with the Crew Return Vehicle (CRV), and prohibit access by the Russians from Baikonur by eventually lowering the inclination below $51.6^{\circ}$. However, tether systems may now have relief since ultra-precision microgravity experiments have halted on ISS, the CRV is no longer planned, and the Russians are building a launch site in the French Guiana ( $5^{0} \mathrm{~N}$ latitude) $)^{5,6.7}$. Moreover, another improved TRS was conceived that has a tether passthrough at the ISS CM (extends above and below the ISS as illustrated in Fig. 4) with only a mechanical attachment and operates without using ISS power. That system operates with a control node at or near the top of the tether that uses solar arrays to collect the solar energy and flywheels for energy storage and discharge. Also at this control node is a cathode that expels electrons to the ionosphere. The electrons were originally collected by an anode near the bottom of the tether. As with all EDT, the power is used to "pump" electrons up the tether, which creates a current in the presence of the Earth's magnetic field causing a thrust tangential to the orbital motion ${ }^{8}$.

The tether pass-through at the ISS CM has a roller and mechanical gear to drive the tether up or down relative

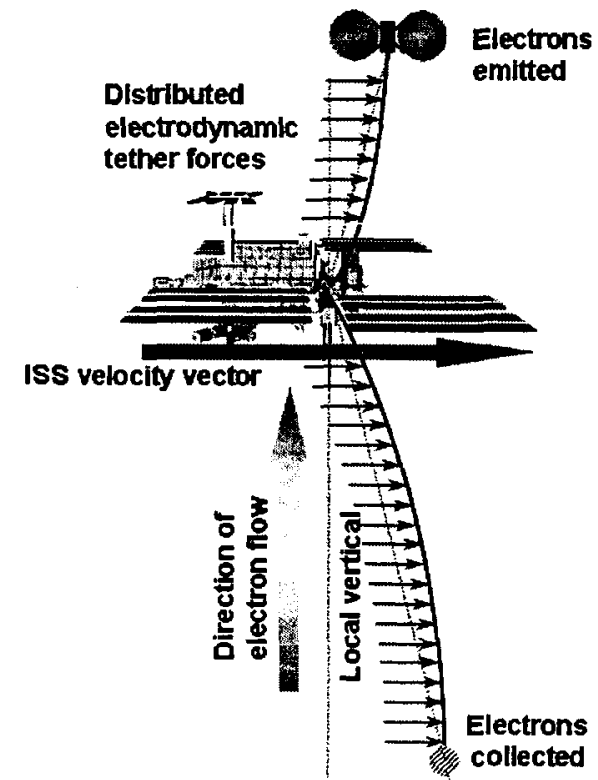

Figure 4. Tether Reboost System Pass-through Concept to the Station. This "Tunable CM" TRS provides complete orbital control by eliminating or reducing Control Moment Gyro (CMG) requirements and allowing gyro de-saturation without propellant. It also increases ISS safety by reducing the amount of propellant that must be delivered, transferred, and stored for rcboost; añu the risk invoived with docking the ISS to highly explosive propellant tank stages. Yearly drag makeup propellant savings are estimated at over $\$ 150$ Million, or nearly $\$ 2$ Billion over a ten-year cycle; funding that could be better spent launching ISS research resupplies, crew and provisions, repair parts, and new exploration related hardware9. Additionally, this same tether design provides the capability to control or "tune" the CM location to a specified location if microgravity experiments once again become prominent on Station. The pass-through design of the TRS will aiso serve a secondary function as a passive gravity-gradient stabilizer. While most Station reboost studies only focus on the drag make-up propellant, the RCS thrusters, which serve to desaturate the gyros also consume large amounts of

This material is declared a work of the U.S. Government and is not subject to copyright protection in the United States

4 
propellant, on the order of a metric ton per year ${ }^{10}$. This inherent tether gravity gradient stabilization naturally provides additional safety in the event of gyro failure (this is additive to the benefit of flywheels serving the backup gyros function), presently a significant ISS safety risk. Similar mass saving benefits as mentioned for the drag makeup case are obtained.

With a TRS on the ISS, the inclination will migrate towards the equator, albeit very slowly when special control algorithms are employed. As previously mentioned, this was a disadvantage of any TRS. With the new Russian launch site in French Guiana, it is a cost savings for the ISS to assume a lower inclination near $28.5^{\circ}$. However, the amount of propellant required to change the inclination with chemical propulsion is extremely high. In contrast, the TRS could intentionally be made to "tug" the ISS into a lower inclination orbit while also reboosting the station without the consumption of propellant or large quantities of power (note in the idealized case where electrical resistive losses are neglected, no net power is required)! At least the option is available to significantly enhance the effect, depending on the particular orbital control algorithm/scheme used in the tether reboost operation mode.

Although there has been no direct system study funding for a station tether over the past years, a synergistic technological program, Momentum-eXchange Electrodynamic Reboost (MXER) tether, has funded and advanced many of the hardware technologies that are required for TRS. MXER is a high-thrust, propellantless propulsion system operating in LEO that can inject human or robotic payloads into higher orbits similar to a chemical upper stage rocket. It is a reusable, long-term infrastructure that can provide roughly one third of the total delta- $\mathrm{V}$ required for typical missions ${ }^{11}$. The MXER tether program has invested in developing a multi-strand aluminum cable with a coating that protects it from Atomic Oxygen (AO) and UltraViolet (UV) radiation, while the multi-strand configuration allows for multiple impacts from MicroMeteoroid and Orbital Debris (MMOD). Glenn Research Center, with funding from the MXER program, has been developing a flywheel model that can drive current (30-40 Amps) into the tether plasma back emf for MXER electrodynamic reboost requirements, which are significantly more demanding than those of a TRS for ISS (2-3 Amps). An automatic jettison system has been preliminarily evaluated in association with MXER development, but more funding and development is needed in this area. Two TRS subsystems that have not received funding and advancement include the mechanical attachment for the TRS to isS and man-rating the TRS propulsion system. The funding needed for advancing TRS subsystems is small and synergistic with other technologies currently being developed. In contrast, the benefits to ISS of using a TRS, even if only in a reduced role, are outstanding and at the same time builds ISS's relevance to the Exploration Initiative.

\section{Exploration And Future Applications}

The revisiting of EDT is not merely a suggestion for an ISS cost savings measure or interesting space experiment. Coupled with other technologies and recent events, it may be a stepping-stone to meeting the long-term architecture requirements of a permanent space infrastructure. The potential applications are as many and varied as are the possible paths to developing space. The critical ones believed to be the most efficient and universally applicable are briefly described here. Details and a full explanation of their future applications are expected in dedicated technical papers within the coming year. This proposed ISS application is only a beginning to these wider technology applications that are supplying the technology base, infrastructure, expertise and resources upon which Exploration may draw in the future.

The most important and near-term application of tether technologies in general for exploration purposes is in a single launch "variable gravity" space facility, something ISS is not capable of accomplishing directly. The availability of such a variable gravity test facility would be of great value to future human lunar and Mars missions, while being complementary to ISS. Experiments on the physiological effects of extended periods of time at $1 / 6^{\text {th }}$ and $1 / 3^{\text {rd }} \mathrm{g}$ would provide invaluable knowledge that must be gained prior to sustained surface operations on the Moon or Mars ${ }^{1}$. A tether-based design is clearly the simplest, most inexpensive and fastest method of obtaining that data. Furthermore, the entire spacecraft design philosophy for trips to Mars and beyond by humans will require the biomedical understanding of gravity, or substantial spacecraft structural over-design will ultimately be required (i.e., design everything for a spinning $1 \mathrm{~g}$ artificial environment, instead of some lesser $\mathrm{g}$ value, which will have an exponential effect of reducing the size and mass of the vehicle).

A comparable function to ISS reboost discussed here is the proposed electric propulsion test bed onboard Station. This system would be an experimental platform mounted to the exterior of ISS and serve as a universal electric engine test stand for high power thrusters typical of exploration class missions. Although a variety of engineering alternatives have been proposed, the tethered-bistem boom platform avoids all of the most difficult practical and safety concerns of high power thruster operation in close proximity of Station components (i.e., plume impingement, propellant contamination, electromagnetic interference, static charge buildup, catastrophic thruster failure). Furthermore, this concept has been put forth with the GRC flywheel power modules, similarly described 
here, as the only practical method of providing the peak power such thruster concepts will demand on orbit. Present and future battery concepts, or even capacitors, have not been shown to have near the same power density as the flywheel, without which the test runs would be severely limited. This test bed may be the single most important activity to ISS in terms of long-term relevance to NASA's exploration needs.

An important lunar architecture option exists for exploiting tethers and flywheels in a unique way, even if being a noteworthy delta from the ISS propulsion technology. To send resources from the lunar surface, a rotating mechanical system can provide the orbital energy necessary to escape the lunar surface as suggested by Baker and Zubrin or mentioned by Carroll ${ }^{12,13}$. Such a system would be essentially propellantless, simple and compact (particularly when compared to such devices as magnetic levitated mass drivers), be completely reusable and have flexibility in targeting. They could be deployed at the lunar poles to supply propellant depots orbiting around the moon and taking advantage of in-situ hydrogen and oxygen resources. An alternative placement is along the moon's equator where it would be used to supply liquid oxygen to L1/L2 stations or mass (i.e., lunar material) to these locations as counterbalance mass for Forward's and Hoyt's lun-a-vator or similar tether-based, propellantless transportation system as described by Pearson ${ }^{14,15,16}$.

The flywheel power technology subsystems directly feed a number of their own future exploration applications, although without any ED tether component. Drilling, excavation, base Uninterruptible Power Supplies (UPS) and soil processing are necessary to support In-Situ Resource Utilization (ISRU) operations. Many of these require peak power loads that can be conveniently traded with the flywheel power technology. Because flywheel systems can be sized independently for energy and power (energy storage is a function of wheel inertia while power rating relates to motor size), they are an ideal choice to provide peak power. In addition, flywheel life capability is insensitive to the frequency and magnitude of the peaks, which is a significant advantage over electrochemical batteries. In applications such as surface bases and rovers, flywheels can remain dormant for long periods of time and are less sensitive to temperature fluctuations, contamination, and recharge patterns. Thus, they are most likely to be the mainstay power source for sustainable Moon or Mars bases as well as key to tether based transportation.

ED tether re-boost can provide numerous benefits to any large space assets such as the Hubble Space Telescope (HST) in Low Earth Orbit (LEO) or as space tugs ${ }^{16}$. The availability of an ED tether could have been used to place the HST in a high parking orbit, safely prolonging its life until the James Webb Space Telescope (JWST) is available. Also, an ED tether is the only practical means of changing the orbit inclination of large space assets. For example, changing the inclination of the ISS to 28 degrees would require a propellant mass on the order of $300 \mathrm{MT}$, whereas the mass of the tether and supportive systems would be less than $5 \mathrm{MT}$. Conversely, the ED tether can act as a de-orbit device by allowing electrons to flow freely through the conductive tether. This does not require a power supply to drive the current; it actually generates power. The disposal of dead space assets is now a requirement of space vehicles. Satellites often continue to operate until they have spent their on-board propellant and are then left to drift. A tether attachment can be used for a fast, propellantless controlled de-orbit maneuver.

\section{Conclusions}

It is evident these two technologies are near-term hardware solutions to the International Space Station which will have tremendous impact to its operations and future relevance. The proposed tether-flywheel capability will provide propellantless drag make up and dramatically reduce propellant resupply requirements. This will frec up the mass and volume on future resupply missions which is so desperately needed for crew provisions, new research experiments, repair parts, safety gear, hardware updates, tools, equipments and many other necessary items to make ISS a productive and active center. Just as important is electrical power to drive the experiments, equipment and activities. Flywheels efficiently tap the energy not presently utilized by the present battery power system while taper charging. That energy is available for tether reboost and in turn allows the solar arrays to again solar track, thus producing more electrical power. The flywheels are able to store even more power and make it available for greater reboost to overcome the additional drag of the solar panels facing the ram direction.

The proposed system, whether installed as a demonstration experiment or as qualified Station hardware, can yield nearly identical results. Besides the reboost function, it can give a long-term option of plane change to 28 degree or even to equatorial inclination over a span of several years time. This can offer operational and launch alternatives completely impossible today, yet are likely to occur in the future, including Russian and European launches from French Guiana.

Safety is improved by reducing the number of dangerous propellant resupply operations required and by providing a redundant stabilization system. The tether system would have tremendous impact on the entire ISS structure by providing a naturally gravity-gradient stable configuration. This lessens the need for active gyro stabilization and its associated power and maintenance. This means more electrical energy to experiments or other

This material is declared a work of the U.S. Government and is not subject to copyright protection in the United States 
important tasks and more up mass and volume on service launches devoted to science or crew supplies rather than facility hardware repair parts. Another benefit the tether provides is a moment arm on which to desaturate the gyros without the use of thrusters and the associated propellant consumption. Again, this is significant mass savings when viewed from a logistics perspective over the lifetime of operations.

In addition to the direct benefits to ISS, such a hardware demonstration will advance tether and flywheel technology for implementation within NASA's exploration vision. The development will aid in several other important projects that have a synergistic technical base. In all cases, tethers and/or flywheels are the enabling technology that creates a new capability previously unimagined. The most pressing for NASA is a variable-gravity facility in a single launch, capable of long-term human biomedical studies to answer the most fundamental questions of human spaceflight, as well as, lunar and Mars habitation. An electric propulsion test bed is the ideal candidate for ISS to directly support the Exploration vision for the far future. On the Moon, rotating tethers can provide orbit insertion of payloads using only electrical power and a relatively small reusable mechanical system on the lunar surface. Flywheels should play a wide role in lunar and Mars bases for power supplies, in ISRU equipment and on mobile equipment such as rovers. Unique science missions and LEO space assets also benefit from ED tethers and have been widely documented over many years.

All these varied applications and many more indicate the need for the technology to be advanced to the spaceflight stage in the immediate future. ISS benefits are clear and pronounced. Both the tether and the flywheel have been studied for implementation on ISS several times in the past, with the GRC flywheel technology actually near the flight hardware stage today. Considering recent Exploration needs, changing role of ISS and the technology development plans as projected here, further detailed study is warranted into EDT systems for ISS at some level.

\section{References}

${ }^{1}$ Paine, T. O. et al., "Pioneering the Space Frontier - An Exciting Vision of Our Next Fifty Years in Space," The Report of the National Commission on Space, 1986.

${ }^{2} J o h n s o n$, L., Carroll, J., Estes, R. D., Lorenzini E., Gilchrist B., Martinez-Sanchez, M., Sanmartin, J., and Vas, I., "Electrodynamic Tethers for Reboost of the International Space Station and Spacecraft Propulsion," ALAA Space Programs and Technology Conference, Huntsville, AL, AIAA 96-4250, 1996.

${ }^{3}$ Estes, R. D., Lorenzini, E. C., Sanmartin, J., Pelaez, J., Martinez-Sanchez, M., Johnson, C. L., and Vas, I. E., "Bare Tethers for Electrodynamic Spacecraft Propulsion," Journal of Spacecraft and Rockets, Vol. 37, No. 2, March-April 2000, pp. $205-211$.

${ }^{4}$ Oleson, S. R. and Benson, S. W., "Electric Propulsion for International Space Station Reboost," AlAA 37th Joint Propulsion Conference and Exhibit, Salt Lake City, NASA TM-2002-211313, AIAA-2001-3644, 2001.

${ }^{5}$ Brown, C. and Connell, K., "Space Biology Advocates Join Forces in Talks with Capitol Hill," SpaceRef [online news source], URL: http://www.spaceref.com/news/viewpr.html?pid=16410 [cited 16 March 2005].

${ }^{6} \mathrm{Hertz}, \mathrm{C}$. , "Crew Return Vehicle Interior Layout: Design Process and Product," ALAA Space 2003 Conference and Exposition, Long Beach, NASA TM-2002-211313, AIAA-2003-6354, 2003.

${ }^{7}$ European Space Agency, "Closer ties between ESA and Russia," ESA News [online news source], URL: http://www.esa.int/esaCP/SEMBHY71Y3E index 0.html [cited 19 January 2005].

${ }^{8}$ Cosmo, M.L., and Lorenzini, E.D, Tethers In Space Handbook, Third Edition, Marshall Space Flight Center, Huntsville, AL, 1997, pp. 25-27.

"Macias, B., "Cargo Resupply Analysis for the International Space Station, Post Assembly Complete Timeframe," AlAA $38^{\text {th }}$ Aerospace Sciences Meeting \& Exhibit. Reno, 2000.

iv Vas I. E., Kelly, T. J., and Scarl, El A., "Space Station Reboost with Electrodynamic Tethers," Journal of Spacecraft and Rockets, Vol. 37, No. 2, 2000, pp. 205-211.

${ }^{11}$ Sorensen, K., "Momentum eXchange Electrodynamic Reboost (MXER) Tether Technology Assessment Group Final Report," NASA/MSFC In-Space Propulsion Technology Office, Huntsville, AL, 2003.

${ }^{12}$ Baker, D. and Zubrin, R., "Lunar and Mars Mission Architecture Utilizing Tether-Launched LLOX," AIAA 26th Joint Propulsion Conference and Exhibit, Orlando, AIAA-90-2109, 1990.

${ }^{13}$ Carrol, J., "Tether Applications in Space Transportation," Acta Astronautica, Vol. 13, No. 4, 1986, pp. 165-174.

${ }^{14}$ Hoyt, R. and Uphoff, C., "Cislunar Tether Transport System," AIAA 35th Joint Propulsion Conference and Exhibit, Los Angels, AIAA-99-2690, 1999.

${ }^{15}$ Forward, R. "Tether Transport from LEO to the Lunar Surface", 27th Joint Propulsion Conference and Exhibit, Sacramento, AIAA 91-2322, 1991.

${ }^{16}$ Pearson, J., "Anchored Lunar Satellites for Cisiunar Transportation and Communication," The Journal of Astronautical Sciences, Vol. 27, No. 1, pp. 3962, 1979.

${ }^{17}$ Gilchrist, B. E., Johnson, L., and Bilen, S. G., "Space Electrodynamic Tether Propulsion technology: System Considerations and Future Plans," 16th International Electric Propulsion Conference, IEPC paper 99-220, October 1999.

This material is declared a work of the U.S. Government and is not subject to copyright protection in the United States 